\title{
Dual resonance high frequency bandpass filter
}

\author{
Šejla Džakmić $^{1 *}$, Sehabeddin Taha Imeci ${ }^{2}$ \\ ${ }^{1,2}$ Department of Electrical and Electronics Engineering, International University of Sarajevo, Bosnia
}

\begin{abstract}
*Corresponding author: sdzakmic@ius.edu.ba
(C) The Author

2020.

Published by

ARDA.

Abstract

This paper presents a design of a dual resonance high-frequency bandpass filter using the Sonnet EM Simulator. The filter prototype is enhanced by adding the short stub in the center of the design. A parametric study shows the impact of choosing appropriate configuration characteristics. This is a dual resonance bandpass filter design, with center frequencies $7.8 \mathrm{GHz}$ and $9.3 \mathrm{GHz}$. Performed input match $\mathrm{S} \_11$ at first resonance is-28.87 dB and insertion loss $\mathrm{S} \_21$ is -0.05 $\mathrm{dB}$. The obtained results at second resonance are $\mathrm{S} \_11=-8.97 \mathrm{~dB}$ and $\mathrm{S} \_21=-2.74$ $\mathrm{dB}$. Due to its characteristics, this is a high-Q medium bandwidth dual resonance bandpass filter. The design is simulated and planning to be fabricated with 0.36 $\mathrm{mm}$ thickness and dielectric constant $\varepsilon \mathrm{r}=1$. This paper benefits from its compact size and simple geometry over the similar filters found in the literature.
\end{abstract}

Keywords: Dual resonance, Bandpass filter, Quality factor, Sonnet software, Microstrip

\section{Introduction}

For the recent several years, a rapid development and progress in modern wireless communication technologies causes higher requirements for new designs of digital microwave components. In that context, multiband filter design, as one of the most significant component, imposes to the researchers worldwide. The authors from [1] and [2] describe dual resonance bandpass filter design using Defected Microstrip Structure (DMS). It is suggested to integrate two DMSs with short circuit stub to produce high attenuation and good selectivity [1]. The performance can be improved by using dual-mode dual-band bandpass filters with stubs loaded coupled line [2]. The parametric study showed that characteristics and behavior of dual band resonance has a significant ratio of the resonant bands, enabling such filter to be used in many modern wireless communications. Parallel-coupled and vertical-stacked configurations are presented in [3]. It describes resonant characteristics of an SIR and discusses how SIR dimensions are adjusted to have resonances at two designated center frequencies of the dual-band response. An interesting design of bandpass filter is based on Slow-Wave Resonant Cell with Dual-Resonance [4]. String slow-wave effect causes ultra-wide stopband response. Meanwhile, the nested inter-digital defected ground structure (DGS) utilized under the T-stub can allocate dual-resonance with a compact size. Good dual passband filtering, with return losses higher than $20 \mathrm{~dB}$, without any external impedance transformer feeds, is obtained in [5]. According to [6], dual band resonant behavior has a significant ratio of the resonant bands. The proposed filter is based on stub loaded resonator with two half wave open ring resonators. The obtained resonant frequencies are independent and accuracy can be improved by tuning the dimensions of open ring and stub. An inspiring balanced dual-band bandpass filter design is described in [7]. According to the presented stub-loaded theory, the first differentialmode resonance can be independently designed by the loaded stubs, not affecting the second one. Bandwidths

This work is licensed under a Creative Commons Attribution License (https://creativecommons.org/licenses/by/4.0/ ) that allows others to share and adapt the material for any purpose (even commercially), in any medium with an acknowledgement of the work's authorship and initial publication in this journal. 
of the dual band can be independently controlled, as well. The stub-loaded resonators with full-wave simulation from [8] has characteristic resonant frequency properties where even-mode resonant frequencies can be easily controlled, while odd-mode resonant frequencies are fixed. Due to its characteristics, filter configuration composed of lumped elements has few shortages. The have good performance at lower frequencies, but they are difficult to be implemented at microwave frequencies. Reference [9] suggests a few techniques for lumped to microstrip type filter conversion, suggesting ABCD Network Parameters Method, Image Parameter Technique, and most common conversion method using Shunt Short/Open Circuited Series Stubs. A comparison of filter designs with three and four transmission zeros showed that selectivity will be significantly improved if fourth zero can be achieved. The concept of transmission zero resonator pair (TZRP) is described as a novel method to induce a passband [10]. Passband filter is designed by implementing proximity coupling between TZRPs, obtaining good selectivity and large band rejection. Combined (mixed) electric and magnetic coupling (MEMC), based on two capacitor and one inductor, is employed in lumped element dual-resonance resonator filter design [11]. A dual band bandpass filter is designed with two independently tuned passbands. Transmission zeros placed around the passbands significantly improved the filter performance. An improved circuit performance of bandpass filter can be achieved by having wide stopband response. However, the problem appears when poor stopband response happens at higher order resonant frequencies. There are various suggested solutions for this issue [12] - [13]. To avoid the presence of spurious modes, the concept of two filter designs, microstrip parallel-coupled bandpass filter and crosscoupled bandpass filter, is proposed [14]. It is suggested to irregularly distribute higher order spurious frequencies of SIRs over the stopband, without overlapping. The effect of resonant frequency bandwidth on Q factor is described by the authors from [15] and [16]. Both papers have very high quality factor obtained by very narrow bandpass bandwidth, especially in [15], where $Q=1505$. This paper proposes a novel dual resonant bandpass filter design that operates at high frequencies, with mid- bandwidth and $\mathrm{Q}$ factor values. Its precedence, compared to the other existing configurations, is in having compact size and simple geometry.

A parametric study is done in Sonnet EM simulator. A detailed design description and results are elucidated in the following sections.

\section{Circuit theory}

A simple LC bandpass filter equivalent circuit is shown in Figure 1. It consists of serial and parallel resonance circuits, constructed by lumped elements inductor $L$ and capacitor $C$.

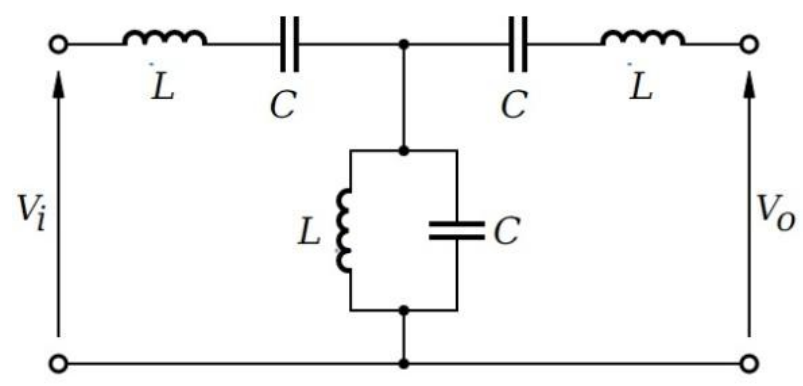

Figure 1. A Butterworth bandpass filter circuit

Bandpass filter can be also constructed by changing the configuration of low pass filter. The transfer function of Butterworth filter is defined by (1):

$$
H(j \omega)=\frac{1}{\sqrt{1+\left(\frac{\omega_{r}}{\omega_{c}}\right)^{2 n}}}
$$

where $\omega_{r}$ is angular resonant frequency $\omega_{r}=2 \pi f_{r}$ and $\omega_{c}$ is angular value of cutoff frequency. Loaded circuit gain is defined by (2): 


$$
\theta=\tan ^{-1} \frac{\frac{1}{L C}-\omega^{2}}{\omega \frac{R}{L}}
$$

A quality factor $Q$ of bandpass filter is a parameter that describes the coupling between the resonant and external circuit, and it is defined by (3):

$$
Q=\frac{f_{r}}{\Delta f}
$$

$\Delta f$ is the bandwidth defined as $\Delta f=f_{2}-f_{1}$, where $f_{1}$ and $f_{2}$ are the frequencies at which the current or voltage magnitude is 0.707 of its value at resonance frequency. Resonant frequency is described by lumped elements:

$$
f_{r}=\frac{1}{2 \pi \sqrt{L C}}
$$

Considering the circuit from Fig. 1, serial and parallel resonance circuits are different in terms of impedance magnitude. Serial resonance circuit has minimum magnitude value of impedance while parallel resonance circuit has maximum value of impedance magnitude, around resonant frequency [17]. Series and parallel resonance circuits of bandpass configuration are defined by (5a) and (5b).

Series resonance circuit:

$$
C_{n}=\frac{B}{2 \pi f_{c}^{2} R_{L} A_{n}} ; \quad L_{n}=\frac{R_{L} A_{n}}{2 \pi B}
$$

Parallel resonant circuit:

$$
C_{n}=\frac{A_{n}}{2 \pi R_{L} B} ; \quad L_{n}=\frac{R_{L} B}{2 \pi f_{c}^{2} A_{n}}
$$

According to the reciprocal dependency of the quality factor and fractional bandwidth form (3), high Q indicates narrow bandwidth and low quality factor refers to the wide bandwidth respectively [18]. Attained quality factor values for suggested filter configuration are shown in next section.

\section{Design methodology and simulation results}

Generally, filters can be constructed in lumped type or microstrip line type. The drawback of lumped type design is that it operates well at low frequencies. This is due to the fact that inductors and capacitors are available for limited frequency range. Moreover, the wavelength of the filter decreases at higher frequencies [9]. Accordingly, it is suggested to design microstrip line circuit, using shunt short or open circuited series stubs for high frequency filter prototypes. A coupled line dual resonance band-pass filter design presented in this paper has configuration shown in Fig. 2. It consists of four ports, each placed at the edge of vertical stubs with $15 \mathrm{~mm}$ length. Vertical stubs are connected by short circuit stub with 33.6 length, all having the width of $1.8 \mathrm{~mm}$. Four vertical stubs are expanded for $45^{\circ}$. Expanded parts of the stubs have $3.8 \mathrm{~mm}$ of length. At the first sight, it is obvious that proposed filter configuration is constructed by two symmetrical configurations, connected together without any gap between them, making the entirety.

In order to meet design specification, many simulations of the proposed filter design are done. This involved many variations applied to the initial configuration. Variations in width of the stubs were firstly applied. Simulations showed that changes in width of the stubs affect the width of band-stop region as well as $\mathrm{S}$ parameter values. Obtained $\mathrm{S}$ - parameters for very small width of the stubs (about $0.8 \mathrm{~mm}$ ) were about 
$S_{11}=0 \mathrm{~dB}$ and $S_{21}=-32 \mathrm{~dB}$ for one resonance. It is also observed that the width of the horizontal and vertical stubs should be equal for the best filter performance.

The configuration performance is enhanced by adding the vertical stub on the center of transmission line. Few variations of the center stub were also to improve the analysis results. Initially, there was a gap between the center stub and transmission line itself, having the width equal to the width of the stubs.

However, this produced many reflections unappreciable parametric study results. Reducing the gap between them caused better filter performance, so the final configuration does not have gap around center stub at all. When the center stub has dimensions of $3.2 \mathrm{~mm}$ width and $4.0 \mathrm{~mm}$ length, design specifications are met.

Beyond mentioned filter configuration variations; air and ground thickness values were also changed and analyzed in order to meet the design specifications. A deeper description of implemented variations is provided in next section, together with the tables of all recorded results for different variations.

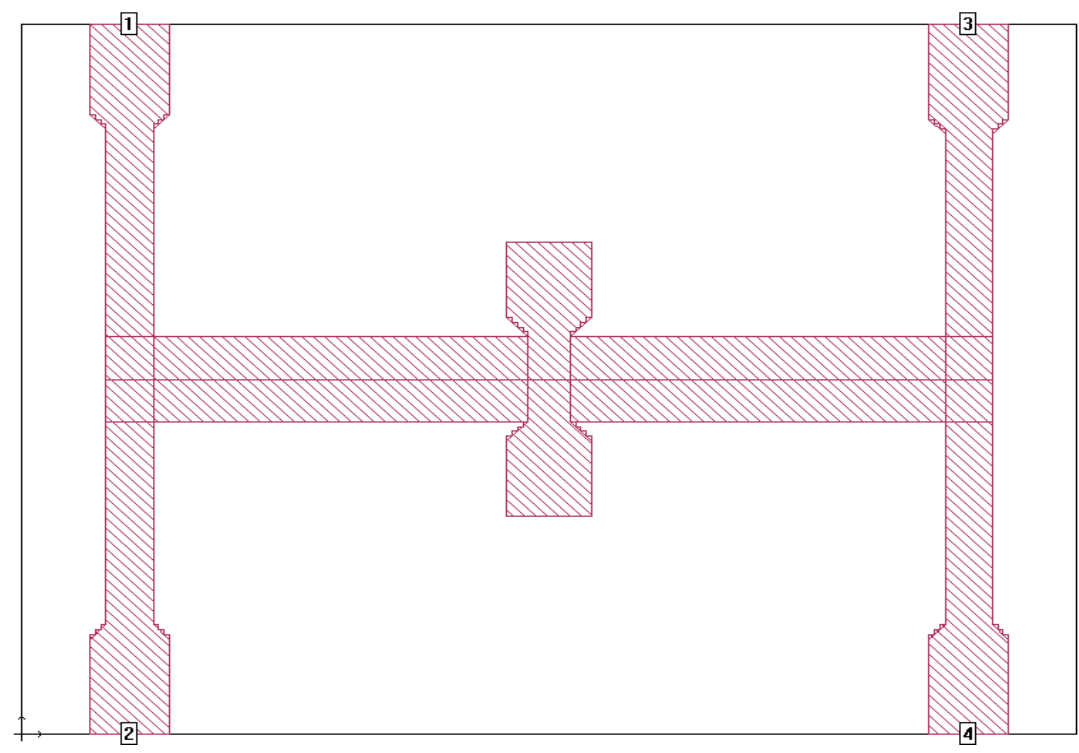

Figure 2. Configuration of the proposed dual band-pass filter

Filter design is finally simulated with a relative dielectric constant of 1 and thickness of the substrate is 0.36 $\mathrm{mm}$. The whole backside of the substrate is the ground plane. Fig. 3 represents a 3D view of filter proposed filter configuration. It is similar to the general structure of a microstrip that contains conducting strip and ground (conducting) plane at the bottom of substrate. The width of the configuration is approximately 34.8 $\mathrm{mm}$ with height $30.0 \mathrm{~mm}$. The filter box has dimensions $55.0 \mathrm{~mm}$ by $30.0 \mathrm{~mm}$.

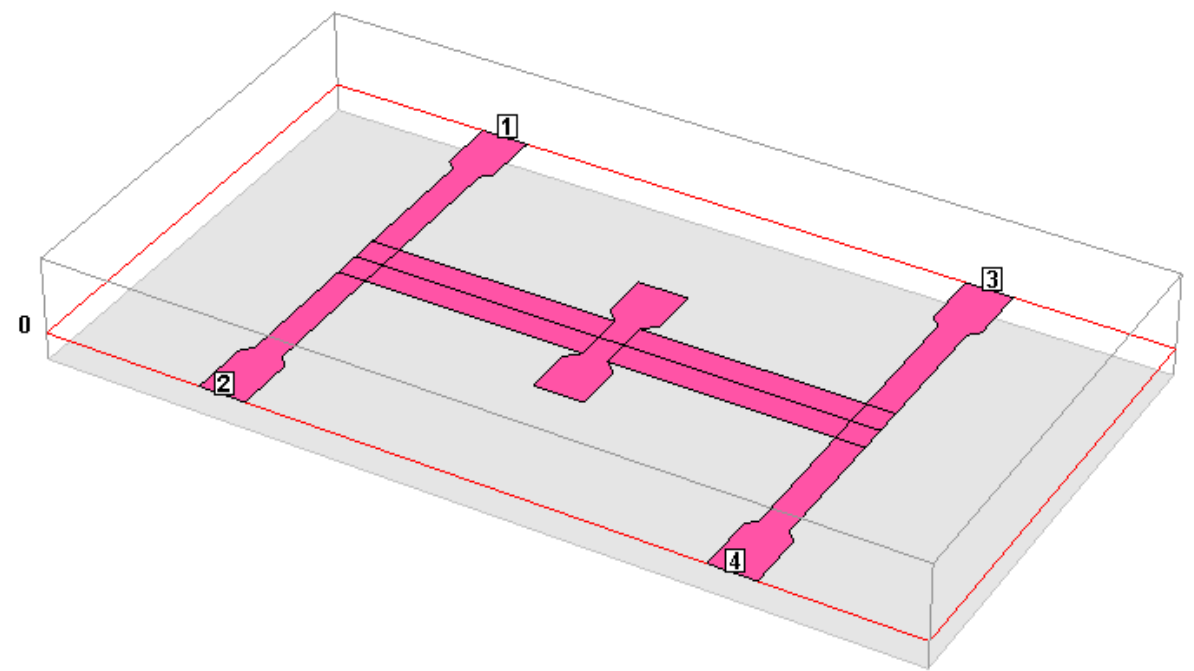

Figure 3. 3D View of the proposed filter configuration 


\section{Parametric study}

For the performance validation, the proposed dual bandpass filter design is simulated with the bandwidth from $6 \mathrm{GHz}$ to $10 \mathrm{GHz}$. To meet design specifications several modifications are applied to the initial prototype. First of all, it is determined appropriate dielectric (air) thickness value.

Table 1 represents the comparison of the results collected for some tested $\boldsymbol{\varepsilon}_{\boldsymbol{r}}$ values. Besides listed values, few others were considered, but those with better performance are recorded in table.

Usually it is suggested to have at least five times bigger dielectric thickness than ground thickness, so other values tested $\varepsilon_{r}$ values were 4, 5, 6,7 and 9. Notwithstanding, this could not be applied to the proposed filter configuration since the obtained results for such specifications were not satisfactory.

Beside this, ground thickness was firstly considered to be 1 or 1.5. After few simulations, it was obvious that final design specifications are met when relative dielectric constant (air thickness) is 1 and ground thickness of $0.36 \mathrm{~mm}$.

Table 1. A comparison of the filter performance for different air thickness

\begin{tabular}{c|c|c|c|c|c|c}
\multirow{2}{*}{ Air thickness } & $f_{1}(\mathrm{GHz})$ & $S_{11}(\mathrm{~dB})$ & $S_{21}(\mathrm{~dB})$ & $f_{2}(\mathrm{GHz})$ & $S_{11}(\mathrm{~dB})$ & $S_{21}(\mathrm{~dB})$ \\
\hline 1.15 & 7.84 & -31.94 & -0.13 & 9.66 & -7.47 & -2.83 \\
1.25 & 7.82 & -32.53 & -0.05 & 9.58 & -14.42 & -2.09 \\
$\mathbf{1 . 0}$ & $\mathbf{7 . 8 6}$ & $\mathbf{- 2 8 . 8 7}$ & $\mathbf{- 0 . 0 5}$ & $\mathbf{9 . 6 4}$ & $\mathbf{- 8 . 9 7}$ & $\mathbf{- 2 . 7 4}$ \\
1.5 & 7.8 & -36.15 & -0.06 & 9.5 & -12.32 & -2.35 \\
1.75 & 7.8 & -32.81 & -0.06 & 9.5 & -12.19 & -2.29 \\
2.0 & -7.78 & -38.12 & -0.061 & 9.46 & -12.12 & -2.29
\end{tabular}

Two resonant frequencies are obtained:

$$
\begin{gathered}
f_{r 1}=7.8 \mathrm{GHz} \\
f_{r 2}=9.3 \mathrm{GHz}
\end{gathered}
$$

Resonant frequency can be also calculated by taking square root of cutoff frequencies from (4):

$$
f_{r}=\sqrt{f_{1} f_{2}}
$$

Passband bandwidths are $\Delta_{1}=25 \%$ and $\Delta_{2}=8 \%$. Parametric study generated four $\mathrm{S}$ parameters for two resonances with $S_{11}$ as input match and $S_{21}$ as insertion loss:

$$
\begin{array}{lll}
f_{r 1}: & S_{11}=-28.87 d B & S_{21}=-0.05 d B \\
f_{r 2}: & S_{11}=-8.97 d B & S_{21}=-2.74 d B
\end{array}
$$


First resonant frequency has the bandwidth of $25 \%$ and second one is about $8 \%$. Referring to (3) from section II, quality factors are found to be:

$$
\begin{gathered}
Q_{1}=\frac{7.8}{0.25}=31.2 \\
Q_{2}=\frac{9.3}{0.08}=116.3
\end{gathered}
$$

A quality factor is a measure of the configuration performance in terms of its losses and resonant bandwidth. In that context, high quality factor values obtained for suggested filter configuration indicate low losses and medium resonance bandwidth. This is also shown in Tables 1-3 where Scattering parameters ( $\mathrm{S}$ - parameters) are listed. Described frequency response is shown in Fig. 4.

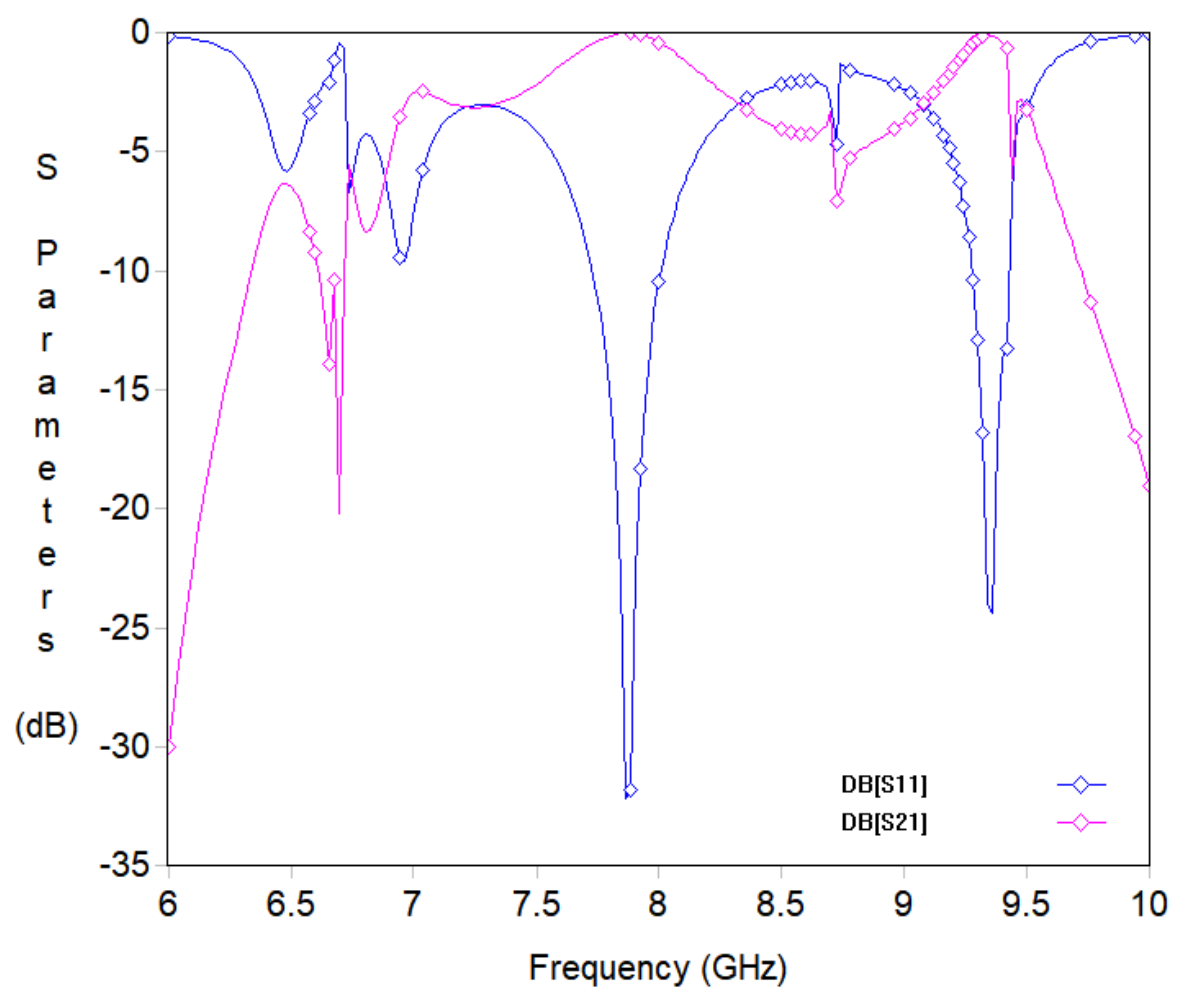

Figure 4. Band-pass filter simulation response

Parametric study from Table 2 and 3 represents the effect of configuration variations. The focus of data provided in those tables was on the center stub dimensions; since it is proved that it has the most impact on filter performance. Many variations of the center stub configuration are applied for best filter performance. A comparison of the filter performance for various dimensions of the center stub highlights the impact of choosing appropriate ones to meet design configurations.

Finally, the proposed design configuration has the center stub with $4.0 \mathrm{~mm}$ length and $3.2 \mathrm{~mm}$ width. Its design is very similar to vertical stubs, being expanded for $45^{\circ}$ at the ends.

Table 2. Configuration performance with different width of the center stub

\begin{tabular}{c|c|c|c|c|c|c}
$\begin{array}{c}\text { Width of the } \\
\text { center stub }\end{array}$ & $\begin{array}{c}f_{1} \\
(\mathrm{GHz})\end{array}$ & $\begin{array}{c}S_{11} \\
(\mathrm{~dB})\end{array}$ & $\begin{array}{c}S_{21} \\
(\mathrm{~dB})\end{array}$ & $\begin{array}{c}f_{2} \\
(\mathrm{GHz})\end{array}$ & $\begin{array}{c}S_{11} \\
(\mathrm{~dB})\end{array}$ & $\begin{array}{c}S_{21} \\
(\mathrm{~dB})\end{array}$ \\
\hline
\end{tabular}




\begin{tabular}{c|c|c|c|c|c|c}
$\begin{array}{c}\text { Width of the } \\
\text { center stub }\end{array}$ & $\begin{array}{c}f_{1} \\
(\mathrm{GHz})\end{array}$ & $\begin{array}{c}S_{11} \\
(\mathrm{~dB})\end{array}$ & $\begin{array}{c}S_{21} \\
(\mathrm{~dB})\end{array}$ & $\begin{array}{c}f_{2} \\
(\mathrm{GHz})\end{array}$ & $\begin{array}{c}S_{11} \\
(\mathrm{~dB})\end{array}$ & $\begin{array}{c}S_{21} \\
(\mathrm{~dB})\end{array}$ \\
\hline 2.2 & 7.88 & -27.51 & -0.02 & 9.42 & -7.98 & -1.89 \\
2.6 & 7.84 & -34.13 & -0.07 & 9.5 & -9.42 & -3.14 \\
$\mathbf{3 . 2}$ & $\mathbf{7 . 8 6}$ & $\mathbf{- 3 4 . 1 2}$ & $\mathbf{- 7 . 7 e - 3}$ & $\mathbf{9 . 2 6}$ & $\mathbf{- 2 0 . 5 9}$ & $\mathbf{- 0 . 4 8}$ \\
2.8 & 7.86 & -34.33 & -0.01 & 9.36 & -27.27 & -0.04 \\
3.0 & 7.86 & -32.89 & $-9.2 \mathrm{e}-3$ & 9.36 & -22.12 & -0.03 \\
3.1 & 7.86 & -36.89 & -0.01 & 9.32 & -23.39 & -0.14
\end{tabular}

Table 3. Configuration performance with different length of the center stub

\begin{tabular}{c|c|c|c|c|c|c}
$\begin{array}{c}\text { Length of the } \\
\text { center stub }\end{array}$ & $\begin{array}{c}f_{1} \\
(\mathrm{GHz})\end{array}$ & $\begin{array}{c}S_{11} \\
(\mathrm{~dB})\end{array}$ & $\begin{array}{c}S_{21} \\
(\mathrm{~dB})\end{array}$ & $\begin{array}{c}f_{2} \\
(\mathrm{GHz})\end{array}$ & $\begin{array}{c}S_{11} \\
(\mathrm{~dB})\end{array}$ & $\begin{array}{c}S_{21} \\
(\mathrm{~dB})\end{array}$ \\
\hline 4.4 & 7.88 & -36.82 & -0.02 & 9.26 & -14.91 & -1.41 \\
4.2 & 7.88 & -34.08 & -0.03 & 9.3 & -19.89 & -0.55 \\
$\mathbf{4 . 0}$ & $\mathbf{7 . 8 6}$ & $\mathbf{- 3 7 . 8 4}$ & $\mathbf{- 0 . 0 2}$ & $\mathbf{9 . 3 4}$ & $\mathbf{- 2 4 . 1 4}$ & $\mathbf{- 0 . 2 3}$ \\
3.8 & 7.86 & -34.66 & -0.01 & 9.36 & -24.66 & -0.09 \\
3.4 & 7.89 & -39.42 & -0.02 & 9.44 & -14.8 & -2.7 \\
3.2 & 7.88 & -32.23 & -0.27 & 9.4 & -11.23 & -2.3
\end{tabular}

\section{Conclusion}

In this paper, a novel dual resonance bandpass filter design is proposed. Filter description is given in details, including the results of various changes applied to the initial filter design. It is designed and optimized in Sonnet EM Simulator [19] to verify the proposed structure. The simulation tests of the suggested configuration led to very successful results, having dual resonances centered at $7.8 \mathrm{GHz}$ and $9.3 \mathrm{GHz}$, with the bandwidths $\Delta_{1}=25 \%$ and $\Delta_{2}=8 \%$ respectively. The performed input match and insertion loss are $S_{11}=$ $-28.87 \mathrm{~dB}$ and $S_{21}=-0.05 \mathrm{~dB}$, at first resonance, and $S_{11}=-8.97 \mathrm{~dB}$ and $S_{21}=-2.74 \mathrm{~dB}$ for the second one, respectively. Due to its characteristics and quality factor values, proposed filter design can be described as a medium bandwidth dual resonance bandpass filter. Throughout the simulations, it is proved to be very efficient and applicable in wireless communications. The future plan is to fabricate proposed filter design on microstrip, measure the results and compare it to the simulation results. 


\section{Acknowledgements}

Special thanks to International University of Sarajevo (IUS) for providing Sonnet Software Licenses.

\section{References}

[1] M. A. Mutalib, Z. Zakaria, N. A. Shairi, S. W. Yik, Y. E. Maskurin, and M. K. Zahari, "Dual-band bandpass filter using defected microstrip structure (DMS) for WIMAX applications," J. Telecommun. Electron. Comput. Eng., vol. 9, no. 1-5, pp. 111-114, 2017.

[2] J. Xu and $\mathrm{W}$. Wu, "Compact microstrip dual-mode dual-band band-pass filters using stubs loaded coupled line," Prog. Electromagn. Res. C, vol. 41, no. May, pp. 137-150, 2013.

[3] J. T. Kuo, T. H. Yeh, and C. C. Yeh, "Design of microstrip bandpass filters with a dual-passband response," IEEE Trans. Microw. Theory Tech., vol. 53, no. 4 I, pp. 1331-1336, 2005.

[4] Z. Zhang, B. Yang, H. Qian, and X. Luo, "Dual-band bandpass filter based on slow-wave resonant cell with dual-resonance," 2016 IEEE MTT-S Int. Conf. Numer. Electromagn. Multiphysics Model. Optim. NEMO 2016, vol. 3, pp. 5-6, 2016.

[5] S. Sun and L. Zhu, "Compact dual-band microstrip bandpass filter without external feeds," IEEE Microw. Wirel. Components Lett., vol. 15, no. 10, pp. 644-646, 2005.

[6] M. F. Hasan, A. S. A. Jalal, and E. S. Ahmed, "Compact dual-band microstrip band pass filter design based on stub loaded resonator for wireless applications," Prog. Electromagn. Res. Symp., pp. 18101814, 2017.

[7] X. Wu, F. Wan, and J. Ge, "Stub-Loaded Theory and Its Application to Balanced Dual-Band Bandpass Filter Design," IEEE Microw. Wirel. Components Lett., vol. 26, no. 4, pp. 231-233, 2016.

[8] X. Y. Zhang, J. X. Chen, Q. Xue, and S. M. Li, "Dual-band bandpass filters using stub-loaded resonators," IEEE Microw. Wirel. Components Lett., 2007.

[9] S. I. s. Hassan and Y. Wahab, "A review of technique to convert low pass filter into microstrip line circuit," in 2008 IEEE INTERNATIONAL RF AND MICROWAVE CONFERENCE PROCEEDINGS, 2008.

[10] Q. Xue and J. Y. Jin, "Bandpass Filters Designed by Transmission Zero Resonator Pairs with Proximity Coupling," IEEE Trans. Microw. Theory Tech., 2017.

[11] J. Xu, W. Wu, and G. Wei, "Novel Dual-Band Bandpass Filter and Reconfigurable Filters Using Lumped-Element Dual-Resonance Resonators," IEEE Trans. Microw. Theory Tech., 2016.

[12] and C.-Y. C. Cheng-Chung Chen, Yi-Ru Chen, "Miniaturized Microstrip Cross-Coupled Filters Using Quarter-Wave or Quasi-Quarter-Wave Resonators," IEEE Trans. Microw. Theory Tech., vol. 51, no. 1, 2003.

[13] W. T. H. J. T. Kuo, W. H. Hsu, "Parallel coupled microstrip filters with suppression of harmonic response," IEEE Microw. Wirel. Compon. Lett., vol. 12, no. 10, pp. 383-385, 2002.

[14] C. F. Chen, T. Y. Huang, and R. B. Wu, "Design of microstrip bandpass filters with multiorder spurious-mode suppression," IEEE Trans. Microw. Theory Tech., 2005.

[15] A. L. Borja, A. Belenguer, H. Esteban, and V. E. Boria, "Design and Performance of a High-Q Narrow Bandwidth Bandpass Filter in Empty Substrate Integrated Coaxial Line at Ku-Band," IEEE Microw. Wirel. Components Lett., 2017.

[16] M. Chose, J. M. Chuma, A. Yahya, O. B. Kobe, I. Ngebani, and T. M. Pholele, "A low-loss 2ndorder chebychev microwave cavity band pass filter," in 2016 IEEE Radio and Antenna Days of the Indian Ocean, RADIO 2016, 2016.

[17] M. Alper Uslu and Levent Sevgi, "A MATLAB-Based Filter-Design Program: From Lumped Elements to Microstrip Lines," IEEE Antennas Propag. Mag., vol. 53, no. 1, 2011.

[18] P. Rizzi, Microwave Engineering: Passive Circuits. 1988. 
[19] "Sonnet Suites, ver. 17.52." [Online]. Available: www.sonnetsoftware.com. 